\title{
Kernos
}

Revue internationale et pluridisciplinaire de religion grecque antique

19 | 2006

Varia

\section{L’objet narratif ou le mythos matérialisé}

Généalogies et catalogues sans paroles au sanctuaire d'olympie

\section{Anne-Françoise Jaccottet}

\section{Q OpenEdition \\ 1 Journals}

Édition électronique

URL : https://journals.openedition.org/kernos/454

DOI : 10.4000/kernos.454

ISSN : 2034-7871

Éditeur

Centre international d'étude de la religion grecque antique

Édition imprimée

Date de publication : 1 janvier 2006

Pagination : 215-228

ISSN : 0776-3824

\section{Référence électronique}

Anne-Françoise Jaccottet, «L'objet narratif ou le mythos matérialisé », Kernos [En ligne], 19 | 2006, mis en ligne le 22 mars 2011, consulté le 24 août 2022. URL : http://journals.openedition.org/kernos/454 ; DOI : https://doi.org/10.4000/kernos.454 


\title{
L'objet narratif ou le mytbos matérialisé Généalogies et catalogues sans paroles au sanctuaire d'Olympie
}

\begin{abstract}
Résumé : Peut-on concevoir un récit généalogique ou une forme catalogique en dehors de tout contexte littéraire? Le sanctuaire d'Olympie offre plusieurs pistes de réflexion à travers l'analyse de ses offrandes et de son décor figuré : généalogie sous-jacente liée à l'identité grecque, agencement parataxique du décor archaïque (coffret de Cypselos), catalogue des douze travaux d'Héraclès, ou catalogue abstrait suscité par un épisode épique. Les différentes stations de cette visite du sanctuaire ouvrent la voie à une réflexion sur la complémentarité - et non l'interdépendance directe - du récit verbal et des représentations figurées. En tant que centre panhellénique le sanctuaire est un creuset dans lequel se met en place une koinè mythologique. Les images participent grandement à cette construction de l'identité culturelle hellénique, aussi bien comme déclencheur du récit, que comme medium à part entière.
\end{abstract}

Abstract: The Narrative Object, or Myth Materialised. Genealogies and Catalogues without Words in the Sanctuary of Olympia. Is it conceivable that genealogical accounts or forms of catalogues, divorced from any literary context, can exist? The sanctuary of Olympia, through an analysis of its votive offerings and figural decoration, yields several paths of reflection: the omnipresence of genealogy, intimately connected with the Greek identity, paratactic arrangements of archaic decoration (the chest of Kypselos), the twelve labours of Herakles, or an abstract catalogue attached to an epic episode. The different stations in this sanctuary visit lead us to reflect on the complementarity - but not direct interdependence - of narrative and figural representation. As a Panhellenic sanctuary, Olympia is a veritable melting pot, in which a mythological koine gradually evolves. Images play a large part in the construction of Hellenic cultural identity: as stimulators of narrative, certainly, but also as media in themselves.

Une mythographie sans texte, est-ce vaine chimère que de l'envisager? Les diverses interventions de ce colloque ont bien montré l'ancrage littéraire des formes catalogiques et généalogiques de la mythographie. Peut-on dès lors concevoir un récit généalogique en dehors de tout contexte littéraire ? Peut-on appréhender une forme catalogique qui s'exprimerait sans texte et peut-être même sans $\operatorname{mots}^{1}$ ? Je crois que certains objets archéologiques sont en euxmêmes une mythographie ou, pour mieux dire, un support mythographique.

\section{Le centaure de Lefkandi : une mythographie sans texte}

L'exemple le plus célèbre en est le centaure trouvé à Lefkandi, sur la côte eubéenne, entre Chalcis et Érétrie (Fig. 1).

\footnotetext{
${ }^{1}$ La réflexion de Claude Calame, dans ce volume, sur les listes, prémisses des catalogues, que l'on retrouve sous forme orale comme sous forme graphique est tout à fait capitale pour la question. On relèvera également, dans le développement de cette problématique, la contribution de Natacha Massar sur les « chroniques » des sanctuaires hellénistiques.
} 


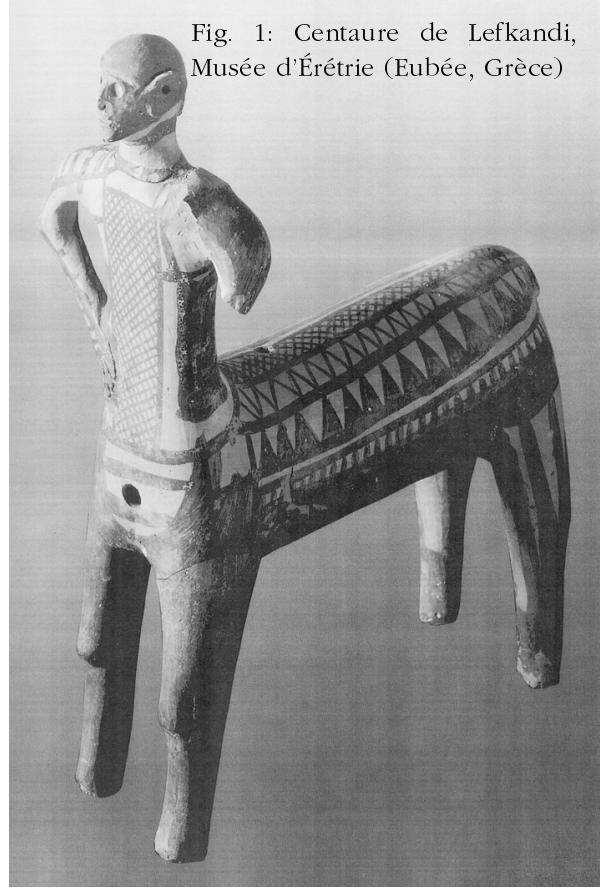

Cette figurine de terre cuite, d'une hauteur de $36 \mathrm{~cm}$, a été découverte dans deux tombes distinctes de la nécropole dite de la Toumba, la tête dans la tombe 1 , le reste dans la tombe $3^{2}$. L'objet lui-même, daté tant stylistiquement que par le contexte des tombes du premier quart du $\mathrm{Ix}^{\mathrm{e}}$ siècle av. J.-C., représente clairement un centaure. Cette pièce tout à fait exceptionnelle a suscité de nombreux commentaires ${ }^{3}$; mais elle n'aurait pas sa place ici si elle ne comportait un détail significatif. On remarque sous le genou gauche du centaure une profonde incision. L'analyse a montré que cette entaille avait été faite par le potier ou coroplathe avant la cuisson. C'est cette incision, manifestement volontaire, qui fait basculer ce centaure anonyme dans la sphère du récit mythologique. Par ce signe plastique, l'artisan ne crée pas un simple centaure, mais un centaure singulier, un centaure auquel on est tenté de donner un nom, Chiron, et à partir duquel on peut raconter un mythos ${ }^{4}$. N'a-t-on pas, résumé dans cette entaille, tout le récit d'Héraclès et de son précepteur Chiron, leur alliance dans la lutte contre les Centaures, la blessure involontaire infligée au bon centaure, que celui-ci ne peut guérir malgré toute sa science et qui finira par l'inciter à donner son immortalité à Prométhée, pour cesser

\footnotetext{
${ }^{2}$ Les fouilleurs n'expliquent cette séparation que par l'importance hors du commun que devait avoir le centaure pour les différents membres de la famille ou de la communauté qui le possédaient. Sur la découverte et les rapports de fouilles, $c f$. V. DesBorough, R.V. Nicholls, M. Popham, "A Euboean Centaur », ABSA 65 (1970), p. 21-30; M.R. POPHAM, L.H. SACKeTT, P.G. Themelis (éds), Lefkandi I. The Iron Age. The Settlement. The Cemeteries, Athens / Oxford, The British School of Archaeology at Athens, 2 vol. 1980, en part. p.168-170 et 344-345, planches 251252; sur le centaure lui-même et son interprétation, $c f$. A LEBESSI, "The Relation of Crete and Euboea in the Tenth and Ninth Century B.C. The Lefkandi Centaur and His Predecessors ", in D. Evely, I.S. Lemos, S. Sherrat (éds), Minotaur and Centaur: Studies in the Archaeology of Crete and Euboea Presented to M. Popham, Oxford 1996, p. 146-153, pl. 50 et 53; I.M. SHEAR, «Mycenaean Centaurs at Ugarit», JHS 122 (2002), p. 147-153, pl. 4, a-b; J.M. PADGETT, The Centaur's Smile. The Human Animal in Early Greek Art, New Haven / London 2003, en part. p. 910, 64-65, fig. 3 p. 8.

${ }^{3}$ Cf. supra n. précédente.

${ }^{4}$ Certains commentateurs émettent des doutes sur l'identité du centaure. Peut-être s'agirait-il de Pholos, lui aussi blessé (cf. M. Gisler-Huwiler, LIMC III, s.v. «Cheiron », p. 237-248, en particulier p. 238 no 1 et p. 247). Peu importe ici; l'entaille sert quoi qu'il en soit d'embrayage au récit.
} 
de souffrir? Certes rien ne prouve définitivement que le récit soit déjà complètement élaboré à cette haute époque, ni que les protagonistes portent déjà les noms par lesquels nous les connaissons quelques siècles plus tard. Qu'importe. Cette entaille pratiquée par un artisan aux environs de 900 av. J.-C., à une époque littéralement préhistorique ou du moins clairement pré-littéraire, doit être interprétée comme un signe mythographique tout à fait manifeste. Par elle, le récit devient possible, elle est le signe visible de l'oralité mythologique, l'expression matérielle du récit.

\section{Le sanctuaire d'Olympie...}

Dans le cadre de ce colloque, je me suis donc posé les questions suivantes : les objets narratifs sont-ils capables de susciter une mythographie sans textes ? Les objets ou les décors figurés, peuvent-ils, en dehors de tout travail littéraire, manifester ou susciter des discours de type généalogique ou catalogique ? Pour donner un cadre circonscrit à cette recherche, j’ai suivi une piste, celle des offrandes et du décor figuré du sanctuaire d'Olympie.

Pourquoi ce choix? D'une part parce que le sanctuaire d'Olympie nous offre une double image: une image textuelle, celle que Pausanias nous transmet par sa très ample description du sanctuaire du II $^{\mathrm{e}}$ siècle ap. J.-C $\mathrm{C}^{5}$; mais aussi une image archéologique, puisque les fouilles menées depuis le $\mathrm{XIX}^{\mathrm{e}}$ siècle nous permettent d'ancrer dans la réalité matérielle nos connaissances du sanctuaire, de ses principaux monuments, de sa chronologie et de quelques offrandes ou décors figurés ${ }^{6}$. D'autre part, et surtout peut-être, parce qu'Olympie est un sanctuaire panhellénique et qu'à ce titre, depuis une haute antiquité, il est un lieu de rencontre entre des ressortissants de cités parfois bien éloignées les unes des autres, un lieu d'échanges entre diverses traditions locales, un lieu d'élaboration d'une sorte de koinè mythologique et culturelle.

\section{... de la généalogie...}

Prenons tout d'abord la question généalogique. Peut-on déceler l'expression de généalogies au travers des offrandes et du décor du sanctuaire d'Olympie? On peut certes remarquer en préambule que le récit généalogique des origines des Éléens et des agônes d'Olympie occupe tout le début du livre V de la Périégèse; mais ce récit ne se retrouve aucunement de façon tangible dans le décor ou les offrandes du sanctuaire. Pausanias ne fait ici que suivre une coutume narrative, un processus discursif, sans référence directe

\footnotetext{
${ }^{5}$ Les livres V et VI de la Périégèse traitent de l'Élide et plus particulièrement d'Olympie. Il a été calculé que la description du sanctuaire d'Olympie représentait le 13\% de l'œuvre totale de Pausanias (calcul de Ch. HABICHT, Pausanias und seine 'Beschreibung Griechenlands', München 1985, p. 16 n. 20, effectué à partir du nombre de pages de l'édition M.H. RochA PEREIRA, Leipzig 1973-1981 [2 éd. 1989-1990]).

${ }^{6}$ Pour un historique des fouilles d'Olympie, cf. H. KYrIELEIS (trad. française d'A. VIREYWALlON), «Les fouilles allemandes à Olympie», in A. PASQUiER (éd.), Olympie. Cycle de buit conférences organisé au musée du Louvre par le service culturel du 18 janvier au 15 mars 1999, Paris, La Documentation française (Louvre, conférences et colloques), 2001, p. 45-74.
} 
avec les monuments qu'il va ensuite décrire. Cet exemple doit nous rendre attentifs à notre position face au texte de Pausanias. La Périégèse est bien un document inestimable qui nous donne accès à nombre de connaissances sur les monuments, les rites et les légendes qui nous auraient autrement échappé. Mais ces connaissances sont naturellement filtrées par la subjectivité de l'auteur - elle-même tributaire des exégètes locaux - et par le travail littéraire qu'il convient de reconnaître avant de vouloir faire de Pausanias une source.

Outre la figure d'Héraclès, fils de Zeus, omniprésente à Olympie, les signes généalogiques sont difficiles à mettre en lumière. Pausanias, en décrivant la statue de Phidias prend la peine de s'arrêter sur les Hôrai et les Charites qui surplombent la tête de Zeus ${ }^{7}$ :

Tout en haut du trône, Phidias a représenté au-dessus de la tête de la statue d'un côté les Charites (Grâces), de l'autre les Heures, trois dans chaque groupe. Ce sont les filles de Zeus selon la tradition épique.

Pausanias justifie ici la présence des Hôrai et des Charites, en cette situation faitière, par le fait qu'elles sont filles de Zeus, du moins dans la tradition épique. Ce petit détail nous permet de voir le rôle que pouvait jouer le décor sculpté. Ces deux groupes de divinités - et plus particulièrement leur position de part et d'autre de la tête de Zeus - suscitent un discours généalogique de la part de Pausanias, et certainement de façon plus générale de la part des visiteurs attentifs du temple. La place de choix occupée par les Hôrai et les Charites dans l'œuvre de Phidias demande implicitement une justification que Pausanias donne par une double référence, à la généalogie et à la tradition épique. Ces deux trios surplombant la tête de Zeus sont ainsi en eux-mêmes les déclencheurs d'un discours généalogique, qui renvoie qui plus est à la poésie épique. On entrevoit déjà les rapports étroits et ambigus que le visuel entretient avec le monde du discours, qu'il soit oral, écrit ou littéraire. Un autre exemple éclairera ce dialogue subtil.

Pausanias s'arrête entre autres à l'offrande faite en commun par le peuple des Achéens (d'Achaïe); elle consiste en une base semi-circulaire comportant les statues de «tous ceux qui se sont présentés au tirage au sort pour aller au combat quand Hector défia un Grec en combat singulier. [...] Sur l'autre socle en face, Nestor est représenté au moment où il a jeté le sort de chacun des concurrents dans son casque »" La scène évoquée est bien connue, il s'agit de l'épisode décrit par Homère dans l'Iliade au chant VII, vers 161 et suivants. Une grande partie de la base ainsi que le socle, ont été retrouvés à quelques mètres au Sud de l'entrée du temple de Zeus. La stratigraphie montre que la base a été érigée peu avant les travaux de terrassement pour la construction

\footnotetext{
${ }^{7}$ Pausanias, V, 11.7 (texte établi par M. Casevitz, traduit par J. Pouilloux, commenté par

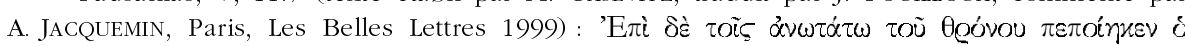

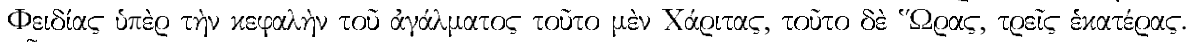

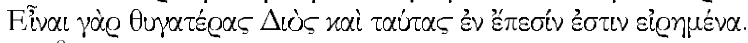

${ }^{8}$ Paus., V, 25.8 (description complète, V, 25. 8-10), trad J. Pouilloux, o.c. (n. 7).
} 
du temple. Elle est donc de peu antérieure à 470 av. J.-C. . Toutes les statues ont bien évidemment disparu; nous sommes donc tributaires de la description de Pausanias pour compléter notre vision de l'offrande. Un détail du texte retiendra notre attention dans la perspective généalogique qui est la nôtre ici. Pausanias remarque que seul Agamemnon porte une inscription l'identifiant de façon certaine. Outre Nestor, reconnaissable à sa position et sans nul doute aux marques de l'âge que le sculpteur n'a pas manqué de transcrire, les huit autres héros de la base semi-circulaire sont difficilement identifiables ${ }^{10}$. Mais le Périégète reconnaît Idoménée, voici comment ${ }^{11}$ :

Le personnage dont le bouclier porte un coq en blason est Idoménée, le descendant de Minos. La famille d'Idoménée descend par sa mère Pasiphaé d'Hélios son père : cet oiseau est, dit-on, l'oiseau sacré d'Hélios, il annonce que le soleil va se lever.

En l'absence d'inscription, Pausanias - ou son indicateur - se fie au langage iconographique pour reconnaître Idoménée. Ce qui nous intéresse ici, c'est que l'épisème du bouclier d'Idoménée mène à l'identification du héros justement par la généalogie : c'est le coq, interprété comme emblème solaire qui permet à Pausanias de retrouver l'ascendance du héros représenté et donc de l'identifier. La généalogie qui s'opère par le biais visuel, iconographique, remplace une inscription identitaire de type verbal. La généalogie d'Idoménée fait partie intégrante de son identité; elle peut même tenir lieu d'identité, comme le montre le raisonnement généalogique de Pausanias. L'identité d'un Grec ne se décline-t-elle pas naturellement par l'énoncé du nom du père, premier pas vers la généalogie?

Ce qui est vrai de l'individu l'est aussi des peuples; et c'est ce que nous apprend encore cette même offrande du peuple d'Achaïe. La dédicace en est en effet la suivante ${ }^{12}$ :

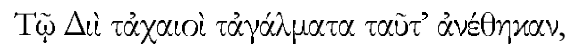

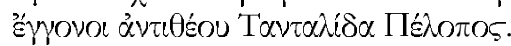

À Zeus les Achéens ont dédié ces statues, eux les descendants du Tantalide Pélops, rival des dieux.

\footnotetext{
${ }^{9}$ Cf. F. ECKSTEIN, ANAOHMATA. Studien zu den Weihgeschenken strengen Stils im Heiligtum von Olympia, Berlin 1969, p. 27-32.

${ }^{10}$ Une des statues manque du temps de Pausanias; il s'agirait d'Ulysse, emporté par Néron à Rome (V, 25. 8). Pausanias tient assurément le renseignement de son guide. On peut toutefois se demander comment Néron ou ses sbires ont reconnu Ulysse parmi les neuf héros; s'il n'y avait pas d'inscription sur la statue (ce qui n'est pas certain), quel signe iconographique permettait l'identification du héros?

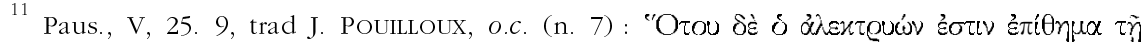

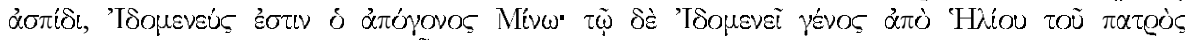

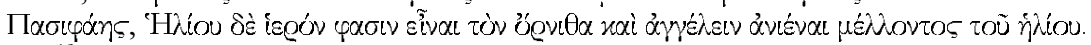

${ }^{12}$ Paus. V, 25. 10, trad J. Poullloux, o.c. (n. 7)
} 
Le peuple d'Achaïe se réclame d'un ancêtre prestigieux et tient à souligner clairement cette ascendance dans la dédicace de l'offrande monumentale qu'il offre à Olympie. L'identité du peuple d'Achaïe passe donc par la généalogie. Mais s'il tient à affirmer son identité littéralement péloponnésienne, le peuple d'Achaïe se rattache, plus subtilement, au passé glorieux des Grecs en général. En se nommant Achéens, les habitants d'Achaïe jouent sur les mots et tissent un lien lexical entre leur identité «nationale» et le terme consacré pour désigner les Grecs dans l'épopée. Ce jeu de mots ne saurait être anodin sur une représentation d'un épisode du cycle troyen! La représentation figurée vient donner corps au jeu lexical de la dédicace. Il s'agit bien pour le peuple d'Achaïe de signifier à la fois son ascendance héroïque péloponnésienne et de revendiquer à son propre compte le passé héroïque et épique de la Grèce.

On voit ici l'importance des développements généalogiques dans la pensée grecque et l'ampleur des procédés d'appropriation identitaire que celles-ci permettent. L'identité grecque est naturellement fondée sur la généalogie qui s'énonce aussi bien verbalement qu'iconographiquement. Un seul signe, comme le coq du bouclier d'Idoménée, peut engendrer un discours généalogique très développé; la seule présence des Hôrai et des Charites sur la statue de Zeus suscite une réflexion généalogique de la part du spectateur attentif. Mais pour être intelligibles, ces signes visuels font appel à une connaissance détaillée des récits généalogiques ou plus généralement des cycles épiques. On voit bien ici l'enchevêtrement du visuel et du verbal, leur complémentarité essentielle. Le signe iconographique est à la fois déclencheur et support du récit généalogique.

Le récit généalogique se trouve dès lors partout et nulle part sous forme embryonnaire. Processus identitaire et culturel essentiel, il est partout, le plus souvent sous-jacent, prêt à surgir d'un infime détail, au gré de l'attention et des connaissances du spectateur/visiteur. Le sanctuaire d'Olympie est un lieu privilégié où étudier ce phénomène au vu de son caractère panhellénique : lieu hors-cité, dont personne ne peut se targuer d'être citoyen, le sanctuaire panhellénique est en effet un lieu identitairement neutre dans lequel il est nécessaire et efficace d'afficher sa propre identité, d'individu comme de peuple; identité qui ne saurait s'exprimer sans référence tacite ou explicite à la généalogie. La pensée généalogique, en tant que procédé identitaire fondamental chez les Grecs, trouve dans un sanctuaire panhellénique, comme Olympie, une place naturelle, peut-être même inconsciente. C'est une certaine ambiance généalogique qui se dégage du sanctuaire bien plus qu'une expression concrète et travaillée du récit généalogique.

\section{... au catalogue}

Qu'en est-il maintenant de la forme catalogique à Olympie. Assurément est-elle plus facile à mettre en évidence; et ce en partie au moins de par les spécificités du système décoratif archaïque et sévère. Prenons d'emblée un exemple très célèbre : le coffret de Cypsélos. Nous n'avons certes pas cette 
offrande corinthienne des environs de 600 av. J.-C., déposée dans l'opisthodome de l'Heraion d'Olympie, du moins au II e siècle ap. J.-C. Là encore c'est grâce à Pausanias et à sa description détaillée que nous pouvons nous faire une idée du décor figuré de ce larnax ${ }^{13}$. Sans rouvrir ce volumineux dossier, nous nous bornerons à souligner l'agencement caractéristique du schéma figuratif. Le décor, fait d'incrustations d'or et d'ivoire sur le corps du coffre, lui-même en bois de cèdre, est réparti en cinq bandeaux qui courent semblet-il autour du coffre. Chacune de ces bandes est divisée en nombreuses scènes juxtaposées les unes aux autres et probablement séparées les unes des autres par une ligne verticale. C'est ce même système parataxique avec séparation graphique que l'on retrouve par exemple sur les brassards de boucliers argiens. Cette parataxe figurative est transcrite, dans la description

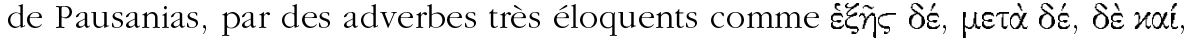
$\delta \dot{\varepsilon}$. Ces adverbes sont la marque verbale et textuelle des signes visuels qui marquaient à n'en pas douter la séparation entre les différentes scènes représentées. L'exposé même du Périégète, par sa structure parataxique claire, évoque lui aussi la discontinuité schématique du décor, réparti en scènes clairement distinctes qui se suivent sur un même bandeau. Cette parataxe de scènes mythologiques différentes est très proche structurellement d'un catalogue mythographique, poétique ou littéraire. Là encore, expression figurée et expression verbale ou littéraire se révèlent fort analogues. Cette proximité est encore renforcée, dans le cas du coffret de Cypsélos, par la présence sur les scènes, non seulement d'inscriptions permettant l'identification des personnages et donc des scènes évoquées, mais encore et surtout par l'ajout ponctuel d'hexamètres explicitant telle ou telle scène. La complémentarité des deux procédés se lit par exemple dans le passage suivant, décrivant le second bandeau depuis la gauche ${ }^{14}$ :

Une femme y est représentée portant sur son bras droit un enfant blanc endormi et sur le gauche un enfant noir semblable à un enfant qui dort; tous les deux ont les pieds divergents. Les inscriptions l'expliquent bien, mais on peut comprendre même sans inscription que ce sont Trépas et Sommeil et que Nuit est leur nourrice commune. [... | Viennent ensuite deux autres femmes au moment où, pilon en main, elles touchent aux mortiers; apparemment elles sont expertes en poison, puisqu'il n'est inscrit à leur propos rien qui permette une autre explication.

\footnotetext{
${ }^{13}$ Paus., V, 17. 5 - 19. 10. Cf. P. ex. R. SplitTer, Die "Kypseloslade" in Olympia. Form, Funktion und Bildschmuck: eine archäologische Rekonstruktion, Mainz, Philipp von Zabern, 2000, avec bibliographie.

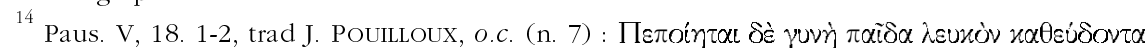

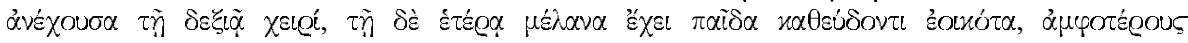

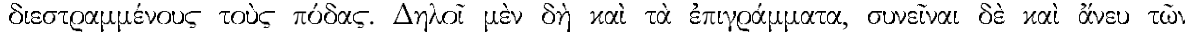

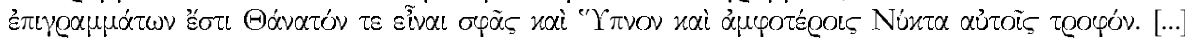

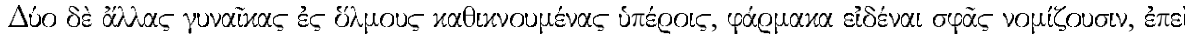

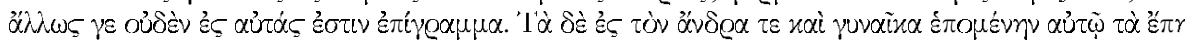

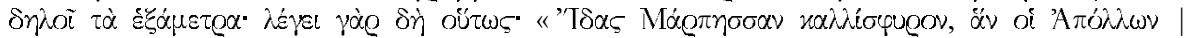

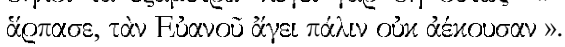


$\mathrm{Au}$ contraire, des vers épiques concernent l'homme et la femme qui le suit. En voici la teneur :

«Idas ramène Marpessa aux belles chevilles, qu'Apollon lui avait ravie, la fille d'Evénos et ce n'était pas malgré elle. »

Les inscriptions identitaires ou les hexamètres ne sont peut-être pas toujours là où ils seraient les plus nécessaires, du moins aux yeux de Pausanias; le témoignage précis du Périégète nous dévoile cependant une particularité du coffret très intéressante pour notre propos. Composé de séquences narratives figurées placées les unes à côté des autres, d'inscriptions servant à identifier les protagonistes, mais encore de vers hexamétriques faisant la synthèse de certains épisodes, le décor du coffret de Cypsélos se place très exactement à l'intersection du figuratif et du littéraire, au croisement de la tradition iconographique et du cycle épique. Seul alors le manque d'unité thématique empêche de faire de ce décor un véritable catalogue mythographique.

Si maintenant nous poursuivons ailleurs dans le sanctuaire la forme parataxique du décor, tout en cherchant l'unité thématique qui nous conduirait à un véritable catalogue, c'est vers le décor du temple de Zeus qu'il convient de nous diriger. Les temples doriques, en effet, par leur frise caractéristique de métopes et de triglyphes alternés, représentent l'exemple le plus manifeste de schéma parataxique dans l'architecture : c'est le rythme architectural qui dicte ici la forme parataxique du décor. Or les deux groupes de six métopes des frises du pronaos et de l'opisthodome du temple de Zeus forment un ensemble thématique très clair. Y sont en effet représentés, en deux cycles complémentaires de six, les douze travaux d'Héraclès. Le témoignage de Pausanias se laisse comparer aux fragments de métopes retrouvées (Fig. 2) ${ }^{15}$ :

On trouve aussi à Olympie la plupart des exploits d'Héraclès. Au-dessus de la porte du temple, il y a la chasse du sanglier d'Arcadie, l'épisode chez le Thrace Diomède, ainsi qu'à Erytheia contre Géryon, ainsi que le moment où il va recevoir la charge d'Atlas et où il va purifier le sol éléen du fumier. Au-dessus de la porte de l'opisthodome, on le voit dérober la ceinture de l'Amazone, on voit l'épisode de la biche, celui du taureau à Cnossos, celui des oiseaux sur le lac Stymphale, celui de l'hydre, ainsi que celui du lion de l'Argolide.

Pausanias omet certes l'épisode de Cerbère au-dessus du pronaos, pourtant bien attesté par les fragments de métope. Mais c'est bien d'un catalogue des douze travaux d'Héraclès qu'il s'agit. Les métopes sont même la première représentation connue du cycle des douze travaux, peut-être même la

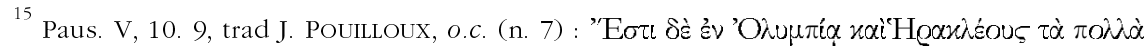
$\tau \tilde{\omega}$ हैG

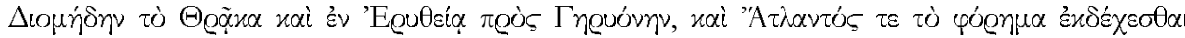

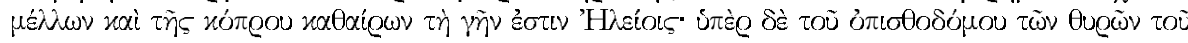

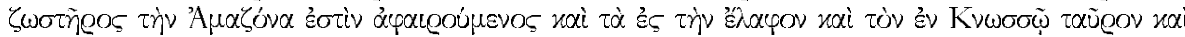

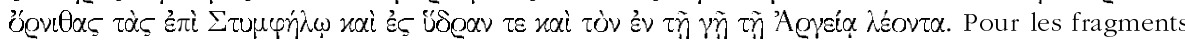
de métopes et leur reconstitution, $c f$. J. BOARDMAN, LIMC V, s.v. "Herakles IV. Herakles' Labours: A. Dodekathlos », p. 7, $\mathrm{n}^{\circ} 1705$ (bibliographie) et p. 8 (dessin).
} 
première mention assurée du nombre canonique de $12^{16}$. Plusieurs indices rendent tout à fait envisageable l'hypothèse selon laquelle les travaux d'Héraclès, présentés dans un cycle fermé de douze épisodes, auraient pris naissance à Olympie. La présence de l'épisode d'Augias, roi d'Élée, est un des arguments en faveur de cette origine éléenne du cycle. Seul Pindare mentionne cet épisode, justement dans une Ode olympique ${ }^{17}$. L'ancrage local de l'épisode d'Augias, à cette époque, montre pour le moins une volonté éléenne de lier la généalogie éléenne aux exploits du prestigieux héros.

On en vient dès lors à se demander si la fixation du nombre de travaux à douze, outre le caractère sacré du nombre en lui-même, ne serait pas liée à des critères architecturaux. La frise dorique du temple de Zeus, composée de deux fois six métopes en façade, en référence tant au panthéon olympien qu'à un module architectural harmonieux, n'est-elle pas la cause ou le facteur déclencheur de la sélection des douze exploits ? N'est-ce pas, finalement à un agencement architectural, puis à la notoriété du sanctuaire et notamment du temple de Zeus, que l'on doit la fixation des douze travaux d'Héraclès, dont le catalogue se perpétuera, pratiquement immuable dans sa composition, durant toute l'Antiquité ? Aucune preuve définitive ne peut être apportée. Mais la question mérite au moins d'être posée.

Quoi qu'il en soit de ses origines, le cycle des douze travaux d'Héraclès à Olympie est bien un catalogue; il apparât même à nos yeux comme un double catalogue : l'original iconographique d'une part - les sculptures des métopes -, et sa transcription textuelle - la description de Pausanias. On voit bien comment la parataxe assez sèche du Périégète, articulée par des roí et des $\tau \varepsilon$, reprend l'ordonnancement des reliefs, isolés les uns des autres par les triglyphes. La mise en parallèle de ces deux catalogues révèle en outre de façon particulièrement patente la fonction des images par rapport à un récit mythographique de forme catalogique. Face aux reliefs, pour lesquels le sculpteur a chaque fois choisi un moment clef à représenter - un moment de l'action elle-même ou le résultat de cette action (le lion de Némée, les oiseaux du lac Stymphale, les pommes d'or des Hespérides) -, Pausanias, lui, se réfère le plus souvent à une sorte de titre résumant l'épisode; la lecture des images passe par une référence au récit, référence orale ou écrite, peu importe ici. Pausanias peut préciser notamment les noms des personnages et les lieux des différents exploits: le sanglier d'Arcadie, le Thrace Diomède, Géryon à Érytheia, le sol éléen, le taureau de Cnossos, le lac Stymphale, le lion d'Argolide. Aucune inscription ne vient préciser ces données sur les métopes (seraient-elle d'ailleurs lisibles du sol ?).

${ }^{16}$ Ce nombre canonique apparaît peut-être antérieurement dans un fragment de Pindare (fr. 169a, v. 43 B. SNell, H. MAEHLER, Pindarus II, Leipzig $1987^{8}$ [1943]. Le texte comportant ] $\varepsilon x \alpha \tau[$, on ne sait si l'épisode mentionné était conçu comme le dixième ou le douzième des travaux. Quoi qu'il en soit, la numérotation que suppose ce fragment montre qu'il existait dans l'esprit de l'auteur une forme de catalogue. Sur l'élaboration du canon des douze travaux, $c f$. J. BOARDMANN, l.c. (n. 15), p. 5-7.

${ }^{17}$ Pindare, Ol. X, 26-30. 

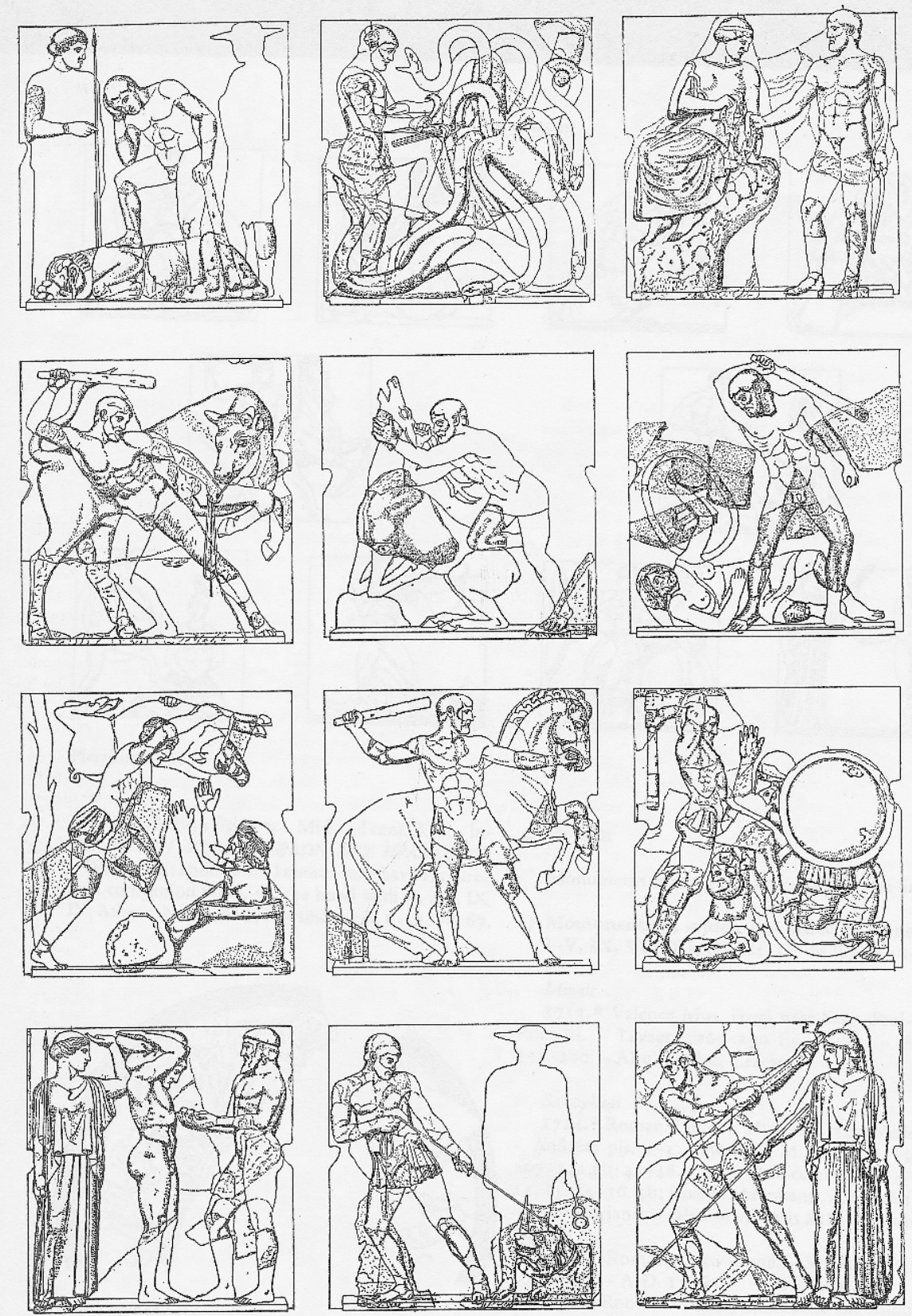

Fig. 2 : Olympie, temple de Zeus, métopes de l'opisthodome et du pronaos, fragments et reconstitution; dessin d'après LIMC V, s.v. « Herakles », n 1705. 
Il est intéressant de comparer brièvement deux épisodes dans leur expression textuelle et dans leur "scénographie » sur les métopes. La ceinture d'Hippolyte tout d'abord: la métope présente Héraclès menaçant de sa massue une amazone au sol, vaincue; Pausanias commente : «on le voit dérober la ceinture de l'Amazone ». Dans l'épisode du sanglier d'Érymanthe, le décalage entre texte et image est tout aussi sensible : Si Pausanias parle de « la chasse au sanglier d'Arcadie », la métope nous présente Héraclès, après la chasse, ramenant sur ses épaules le sanglier vivant, au grand effroi d'Eurysthée qui se réfugie dans sa jarre. Pausanias ne raconte pas les images ellesmêmes, mais ce à quoi elles font allusion. Les reliefs ne sont pas fermés; ils sont des portes ouvertes sur le récit, ils évoquent le récit, le déclenchent. Ils sont une autre expression de la mythographie, une expression qui renvoie à l'ordre du discours tout en concourant à former et à structurer ce discours.

Le rythme architectural est un moule et une structure parataxique qui va influencer de façon indélébile la forme catalogique que prendront les douze travaux d'Héraclès; quant au contenu, la présence inaugurale ici de l'épisode d'Augias, qui deviendra canonique par la suite, montre assez comment les métopes ont pu modeler la matière même du récit catalogique.

Avant de conclure, j'aimerais prendre un dernier exemple de catalogue parmi les offrandes d'Olympie; un exemple qui nous permet de dépasser la parataxe de scènes présentées sur un même plan et séparées entre elles graphiquement - comme sur le coffret de Cypsélos -, ou architecturalement comme sur les métopes du temple de Zeus. Il s'agit d'un groupe sculpté, offert par les Apolloniates et datant de 460/440 av. J.-C. ${ }^{18}$ dont l'archéologie a conservé quelques traces ${ }^{19}$ :

Près du lieu dit Hipodamion, il y a une base de pierre semi-circulaire qui porte des statues de Zeus, ainsi que de Thétis et de Héméra, qui viennent supplier Zeus pour leur enfant. Voilà pour le centre de la base. Dans les gens qui ont l'attitude de combattants, il y a, chacun à une extrémité de la base, Achille et Memnon; selon le même principe sont opposés l'un à l'autre un Barbare et un Grec: Ulysse et Hélénos (car tous deux avaient la plus haute réputation de sagesse dans l'une et l'autre armée), face à Ménélas Alexandre, à cause de leur haine originelle, face à Diomède Enée et face à Ajax, fils de Télamon, Deiphobos. Ce sont des ouvres de Lykios fils de Myron.

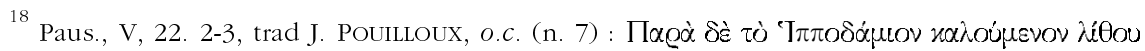

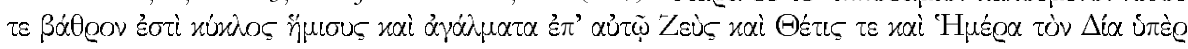

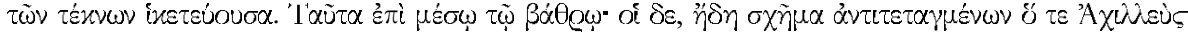

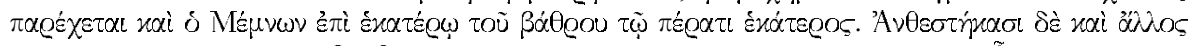

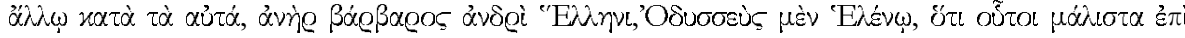

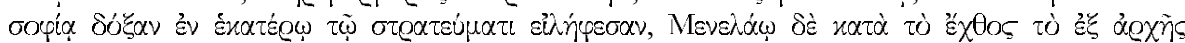

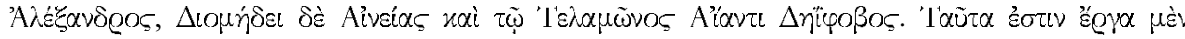
Auxíou toũ Múçuvos. Apollonia, colonie de Corcyre, au Sud d'Epidamne (Dyrrachium) en Illyrie. La date approximative est donnée par le sculpteur, cité par Pausanias, Lykios, fils de Myron.

19 Cf. ECKstein, o.c. (n. 9), p. 15-22: moitié Ouest des fondations (in situ), quatre blocs fragmentaires de l'assise qui portait les statues, deux fragments de la dédicace.
} 
Le thème central de ce groupe sculpté est bien la psychostasie, la pesée des âmes, donc des destins, de Memnon et d'Achille. Nous savons qu'Arctinos de Milet en traitait dans son Ethiopide et qu'Eschyle en avait même fait le titre d'une des tragédies que nous avons perdues ${ }^{20}$. Les représentations du combat d'Achille et Memnon sur le corps d'Antilochos, font très tôt partie du répertoire iconographique ${ }^{21}$. Le coffret de Cypsélos en comportait un exemple; les deux mères étaient représentées, chacune du côté de son fils. Citons encore la frise Est du célèbre trésor des Siphniens à Delphes qui comporte à la fois le combat des deux héros sur le cadavre du fils de Nestor et une assemblée des Dieux, où l'on retrouve les deux mères, Thétis et Éôs (Héméra), avec au centre, dans une malencontreuse lacune, la pesée des âmes ${ }^{22}$. Le sujet de la base des Apolloniates était donc bien connu, tant par le récit, épique ou tragique, que par l'iconographie. Ce qui fait tout l'intérêt ici de ce monument, c'est qu'il ne s'arrête pas aux seuls protagonistes de l'épisode, soit Achille, Memnon, leurs mères Thétis et Héméra (Éôs) et Zeus. Si le trio divin occupe bel et bien le centre de la base, les figures d'Achille et de Memnon sont comme rejetées aux deux extrémités de la base. Entre eux et le trio central sont "intercalés" quatre autres paires de guerriers affrontés l'un à l'autre de part et d'autre de l'hémicycle, un Grec et un Troyen. Or ces couples affrontés ne sont pas forcément directement inspirés d'épisodes épiques. Aucun combat entre Ajax et Deiphobos n'apparaît dans les épopées que nous avons conservées; Ulysse et Hélénos ne semblent pas s'être affrontés; Ménélas et Pâris se livrent effectivement un duel dans l'Iliade ${ }^{23}$, mais celui-ci est interrompu par Aphrodite qui enlève son protégé, Pâris, dans un brouillard; enfin Enée et Diomède se rencontrent bel et bien ${ }^{24}$, mais là encore c'est Aphrodite qui, à grand risque, soustrait son fils à la fureur de Diomède. Que viennent donc faire ces quatre couples de guerriers au milieu de l'épisode du combat d'Achille et de Memnon et de la pesée des âmes qu'il induit? Pausanias s'est sans doute posé cette même question, à laquelle il tente de répondre en mettant en avant les parentés de caractère ou de situation qui rapprochent deux héros : Ulysse et Hélénos sont liés entre eux par « la plus haute réputation de sagesse dans l'une et l'autre armée », alors que Ménélas et Pâris sont appariés «à cause de leur haine originelle ». C'est dans un lien abstrait, mettant en avant des traits de caractères, un peu à la manière des

${ }^{20}$ Respectivement M. DAVIES, Epicorum Graecorum Fragmenta, Göttingen 1988, p. 45-48; S. RADT, Tragicorum Graecorum Fragmenta III, Göttingen 1985, F 279-280a, H.J. METTE, Der verlorene Aischylos, Berlin 1963, p. 110-112.

${ }^{21} C f$. A. Kossatz-Deismann, LIMC VI, s.v. « Memnon », en particulier p. 451-455.

${ }^{22}$ Interprétation de V. BRINKMANN, «Die aufgemalten Namenbeischreiben am Nord- und Ostfries des Siphnierschatzhauses », BCH 109 (1985), p. 77-130, en particulier p. 83-87 et 110-121; cf. également, du même auteur, Beobachtungen zum formalen Aufbau und zum Sinngebalt der Friese des Siphnierschatzhauses, München, 1994.

${ }^{23}$ Homère, Iliade III, 328-382.

${ }^{24}$ Homère, Iliade V, 239-351. 
Vies parallèles de Plutarque, que Pausanias cherche la cohérence de cette offrande monumentale.

Il faut bien constater, avec le Périégète, que l'offrande des Apolloniates est d'une composition intrigante; le « rejet » aux deux extrémités de la base semicirculaire des deux héros concernés par la décision en train d'être prise au centre même du monument montre une volonté de rompre en quelque sorte le récit de l'épisode en intercalant des paires de guerriers qui ne suscitent pas directement le récit, des paires de guerriers qui sont simplement là comme des expressions d'une force de caractère reconnue ou d'un sentiment commun. À l'épisode essentiellement narratif de la psychostasie s'ajoutent des personnages qui représentent de façon relativement abstraite des valeurs. L'épisode de la psychostasie donne lieu à un supplément sous forme de catalogue, comme si l'affrontement d'Achille et de Memnon était prétexte ou stimulus déclencheur à la présentation catalogique de paires de combattants hérö̈ques. Nous ne manquerons pas de souligner dans ce contexte que les

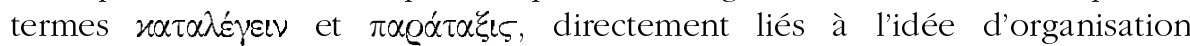
catalogique, sont, chez Homère, les termes techniques traduisant le placement précis et l'organisation des combattants aussi bien dans les rangs que face aux adversaires. La parataxe et le placement catalogique des guerriers dans ce monument du milieu du $\mathrm{v}^{\mathrm{e}}$ siècle av. J.-C. a sans doute encore un lien avec la pratique militaire. Retenons, quoi qu'il en soit, l'apparition sur cette base d'un catalogue « gratuit » venant «s'ajouter » à un épisode bien connu de l'épopée, faisant ainsi cohabiter récit épique et présentation abstraite de qualités morales.

Au terme de cette brève visite des offrandes et du décor figuré du sanctuaire d'Olympie quel bilan peut-on tirer? En premier lieu peut-être doit-on souligner que les grands sanctuaires, les sanctuaires panhelléniques, sont des lieux propices à la réflexion mythographique, de nos jours comme assurément dans l'Antiquité. Ils sont par définition des domaines caractérisés à la fois par l'abondance et par l'hétérogénéité des offrandes, des thèmes, des figures mythologiques; la proximité de telle et telle offrande, de tel et tel sujet mythologique, fruit du hasard plus que d'un procédé raisonné, peut faire naître une envie voire une nécessité de classement. La cohabitation forcée de thèmes figurés souvent fort différents peut faire surgir une classification d'ordre généalogique ou catalogique dans l'esprit du visiteur antique comme dans le nôtre aujourd'hui. Le sanctuaire est comme une liste à l'état brut ${ }^{25}$ : au visiteur d'en saisir le sens, d'y mettre de l'ordre, d'y mettre son ordre, comme le fait Pausanias en donnant un sens, dans toutes les acceptions du terme, à sa visite et à sa description.

Les sanctuaires panhelléniques sont aussi et avant tout un lieu d'échange, une vitrine permettant aux cités, aux peuples mais aussi aux individus de se faire connaître; et qui dit identité, en Grèce antique, dit aussi généalogie. Les

\footnotetext{
${ }^{25}$ Les inventaires d'offrandes dans les sanctuaires mis en lumière dans ce volume par Natacha Massar sont une expression tangible du sanctuaire-liste.
} 
Achéens prennent la peine de se dire descendants de Pélops, sur la base de leur offrande monumentale, alors que le thème choisi, tiré tout droit de l'épopée, marque leur appartenance à la communauté des Grecs. Quant à leur titre d'Achéens, il leur permet de récupérer à leur compte le glorieux passé épique des vainqueurs de Troie. Mais il arrive aussi que le sanctuaire soit une vitrine permettant de présenter des particularismes locaux : le cas de l'épisode d'Augias, qui prend à Olympie, sa place dans ce qui devient le cycle des douze travaux d'Héraclès montre à quel point le sanctuaire peut servir de lieu d'intégration de cycles locaux dans la culture mythologique et mythographique commune. Dans les sanctuaires s'élabore une koinè mythographique à partir certes des grands mythes reconnus de longue date par tous les Grecs mais aussi sur la base de particularismes locaux que les sanctuaires communs ont permis de présenter à l'ensemble des Grecs.

Enfin, d'un point de vue moderne, la profusion du matériel à disposition dans le sanctuaire d'Olympie - littéraire ou figuré - nous permet de réfléchir sur les rapports entre le récit et l'image, entre ce qui se dit et ce qui se montre. Les images ne sont jamais la simple illustration d'un épisode du récit; les récits ne sont jamais la seule traduction en mots de ce que l'on voit. L'image évoque le récit, le déclenche, mais mène à une expérience autre en usant de références qui échappent au domaine verbal; le récit peut s'accrocher à une image mais ira lui aussi dans une direction qui lui est propre, selon des références et un ductus spécifiques. Récit et image ne sont pas directement interdépendants, ils sont deux expressions différentes d'une même idée, deux chemins différents pour exprimer une même pensée, un même univers; traduit en mots ou en images, le monde antique ne s'exprime toujours que partiellement. Le message doit être cherché au-delà des mots, au-delà des images, par la conjonction des expressions verbales et figurées, qui ne sont pas interchangeables ou équivalentes mais complémentaires.

Anne-Françoise JACCOTTET

Université de Lausanne

Institut d'Archéologie et des Sciences de l'Antiquité

BFSH 2

$\mathrm{CH}-1015$ LAUSANNE

Courriel : anne.jaccottet@bluewin.ch 